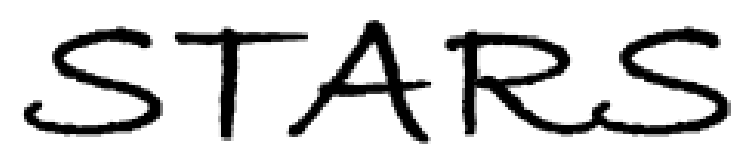

University of Central Florida

STARS

Faculty Bibliography 2000s

Faculty Bibliography

$1-1-2006$

\title{
Two-photon resonance fluorescence
}

Moorad Alexanian

Subir K. Bose

University of Central Florida

Find similar works at: https://stars.library.ucf.edu/facultybib2000 University of Central Florida Libraries http://library.ucf.edu

This Article is brought to you for free and open access by the Faculty Bibliography at STARS. It has been accepted for inclusion in Faculty Bibliography 2000s by an authorized administrator of STARS. For more information, please contact STARS@ucf.edu.

\section{Recommended Citation}

Alexanian, Moorad and Bose, Subir K., "Two-photon resonance fluorescence" (2006). Faculty Bibliography 2000s. 5895.

https://stars.library.ucf.edu/facultybib2000/5895

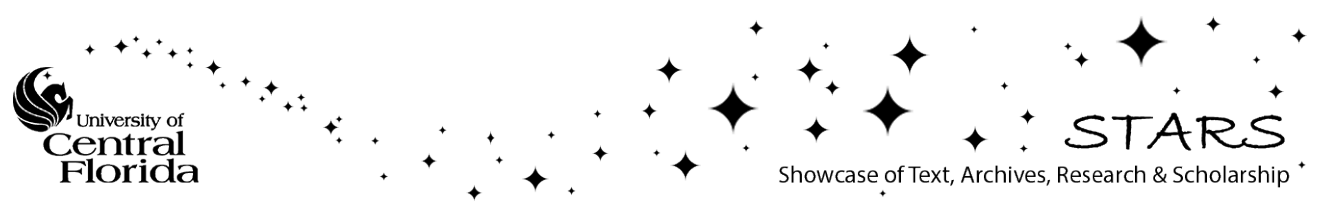




\title{
Two-photon resonance fluorescence
}

\author{
Moorad Alexanian* \\ Department of Physics and Physical Oceanography, University of North Carolina Wilmington, Wilmington, North Carolina 28403, USA
}

Subir K. Bose ${ }^{\dagger}$

Department of Physics, University of Central Florida, Orlando, Florida 32816, USA

(Received 30 June 2006; revised manuscript received 17 November 2006; published 26 December 2006)

\begin{abstract}
We present a theory of two-photon resonance fluorescence of an atom or molecule in which the excitation by an external electromagnetic field as well as fluorescence emission is mediated by two-photon processes. The treatment is based on first dressing the atom or molecule by the external field and then evaluating perturbatively the effect of the interaction with the vacuum or fluorescent field and so resonance fluorescence can be considered as spontaneous emission from the dressed atom. The introduction of the combined system of atom and external field via dressed states leads to simpler calculations and more transparent physics. The fluorescence spectrum derived by us has similarities as well as differences with that of one-photon resonance fluorescence and earlier theoretical predictions for the two-photon case.
\end{abstract}

DOI: 10.1103/PhysRevA.74.063418

PACS number(s): $42.50 . \mathrm{Hz}, 32.80 . \mathrm{Wr}$

\section{INTRODUCTION}

Resonance fluorescence is the response of two-level atoms or molecules to a driving field usually provided by a laser whose frequency is close to the transition frequency of the atom or molecule. Between states of opposite parity, transitions are one-photon, while two-photon processes, dictated by electric-dipole selection rules, mediate transitions between same parity states. In weak fields, an atom would absorb a photon and emit spontaneously in the same frequency. In strong fields, Mollow [1] predicted a three-peak spectrum of fluorescent light, two symmetrically spaced sidebands around a central peak, which is at the same frequency as that of the laser. The sideband frequency depends on the laseratom coupling constant and the average number of laser photons in the quantization volume [2].

Two-photon fluorescence (TPF) is a process in which a chromophore simultaneously absorbs two photons from the laser. The fluorescent light is also mediated by the emission of two photons. TPF has many advantages over the onephoton process, especially in biological applications. A laser of half the frequency of the atomic transition frequency causes less damage to the sample. Imaging with TPF microscopy gives much sharper images than those obtained in onephoton fluorescence microscopy. Denk et al. [3] first demonstrated depth-resolved images of tissues by using TPF. This promising new imaging technique has given rise to a great deal of experimental work in biological systems [4] and as diagnostic tools in biomedical research [5]. TPF microscopy has recently been used to study detection of single molecules, which allow detailed information about the local environment of an individual molecule [6].

In strong fields, the atom-laser field interaction cannot be treated perturbatively, although the effects of the fluorescent field can be treated perturbatively. Compagno and Persico [7]

\footnotetext{
*Email address: alexanian@uncw.edu

${ }^{\dagger}$ Email address: skb@physics.ucf.edu
}

recognized the point and suggested a "dressing" of the atom by the laser field, which can be carried out by a canonical transformation [8]. Fluorescence takes place by transitions between the dressed states of the atom-laser system [9].

In this paper, we present a theory of TPF using the dressed-atom picture of Cohen-Tannoudji [9]. The twophoton interaction between the external driving field and the two-level atom follows from an exact unitary transformation of a three-level atom with single-photon electric dipole transitions only. The unitary transformation decouples the intermediate level from the ground and excited states [10]. The resulting Hamiltonian for the two-level atom gives rise to two-photon transitions between states having the same parity [10]. The dressed-atom approach to TPF, which gives exact results for arbitrary photon states, is particularly suitable for large excitation limits that are more appropriate for experimental realizations. We compare our results with earlier theories of TPF which do not use the dressed-atom approach; in particular, Holm and Sargent [11], Hutchinson and Mavroyannis [12], Cheung [13], Basu et al. [14], and Chen and Freedhoff [15].

\section{TWO-PHOTON HAMILTONIAN}

To study two-photon resonance fluorescence, we consider an effective two-level atom dressed by an external driving field of frequency $\omega$. The Hamiltonian $H_{r}$ explicitly represents two-photon transitions by the occurrence of quadratic photon field operators. $H_{r}$ is given by [16]

$$
H_{r}=\hbar \omega N+E_{0}+\hbar \mu \sigma_{33}+\hbar \eta \sigma_{11}+\hbar \lambda\left(\sigma_{31} a^{2}+\sigma_{13} a^{\dagger 2}\right),
$$

where $a$ and $a^{\dagger}$ are the photon operators of the driving field and $\sigma_{i j}=|i\rangle\langle j|$ are the atomic transition operators. The operator $N=a^{\dagger} a+\sigma_{33}-\sigma_{11}+1$ is a constant of the motion, also called the excitation number. This is an important parameter and high values of the eigenvalues of $N$ would represent high excitation by the driving field. The various parameters $E_{0}, \mu$, 
$\eta$, and $\lambda$ have been obtained in [16] and depend in a highly nonlinear fashion on the operator $N$. In particular, the intensity-dependent couplings are a direct consequence of the exact unitary transformation [10] while previous studies usually introduce them phenomenologically [14].

The Hamiltonian $H_{r}$ is derived from a three-level atom of energy levels $E_{1}, E_{2}, E_{3}$ in ascending order in a cascade configuration in interaction with a single-mode electromagnetic field of frequency $\omega$. The middle level $E_{2}$ is detuned by $\Delta$, where $\Delta=E_{1}-E_{2}-\hbar \omega=E_{3}-E_{2}-\hbar \omega$, and is formally "eliminated" by a unitary transformation such that $H_{r}$ is explicitly two photon [16].

The excited two-level atom can spontaneously emit into the fluorescent modes $(\mathbf{k} j)$. A schematic representation of a resonance fluorescence experiment has been presented by Compagno et al. [17]. In this scheme, a beam of atoms crosses a laser beam at right angles. The atoms absorb and reemit or scatter in all directions into fluorescent field modes, other than the laser mode. The total Hamiltonian, including the fluorescence modes and their two-photon interactions, is given by

$$
H=H_{r}+\sum_{\mathbf{k} j} \hbar \omega_{\mathbf{k}} a_{\mathbf{k} j}^{\dagger} a_{\mathbf{k} j}+\sum_{\mathbf{k} j}\left[\epsilon_{\mathbf{k} j} \sigma_{31} a_{\mathbf{k} j}^{2}+\epsilon_{\mathbf{k} j}^{*} \sigma_{13} a_{\mathbf{k} j}^{\dagger 2}\right]
$$

where $\epsilon_{\mathbf{k} j}$ 's are coupling constants.

The eigenvalues $E_{n}^{ \pm}$and eigenfunctions $\left|\Psi_{n}^{ \pm}\right\rangle$of $H_{r}$ represent mixing or entangling of atomic and photonic states and are called dressed states. One has [16]

$$
\begin{aligned}
& \left|\Psi_{n}^{+}\right\rangle=\sin \theta_{n}|3, n\rangle+\cos \theta_{n}|1, n+2\rangle, \\
& \left|\Psi_{n}^{-}\right\rangle=\cos \theta_{n}|3, n\rangle-\sin \theta_{n}|1, n+2\rangle,
\end{aligned}
$$

where $|1\rangle$ and $|3\rangle$ are the lower and upper atomic states, respectively, and $|n\rangle$ is the photon number eigenstate. Here,

$$
\begin{aligned}
& \cos \theta_{n}=\frac{r(n+2)^{1 / 2}}{\sqrt{n\left(r^{2}+1\right)+2 r^{2}+1}}, \\
& \sin \theta_{n}=\frac{(n+1)^{1 / 2}}{\sqrt{n\left(r^{2}+1\right)+2 r^{2}+1}},
\end{aligned}
$$

with $r \equiv g_{1} / g_{2}$, where $g_{1}$ is the dipole-coupling constant between the ground state level 1 and the intermediate level 2 and $g_{2}$ is the dipole-coupling constant between the intermediate level 2 and the upper level 3 of the three-level atom.

The respective eigenvalues are given [16] by

$$
\begin{aligned}
E_{n}^{+}= & \hbar \omega(n+1)+\frac{E_{1}+E_{3}}{2}-\frac{\Delta}{2} \\
+ & \frac{1}{2} \sqrt{\Delta^{2}+4 \hbar^{2}\left[g_{1}^{2}(n+2)+g_{2}^{2}(n+1)\right]}, \\
E_{n}^{-} & =\hbar \omega(n+1)+\frac{E_{1}+E_{3}}{2} .
\end{aligned}
$$

Note that the dressed states (3) do not depend on the detuning parameter $\Delta$, contrary to a similar treatment of the onephoton resonance fluorescence case [17-19]. However, the energy eigenvalue $E_{n}^{+}$given by Eq. (5) does indeed depend on the detuning parameter $\Delta$ as does the two-photon Rabi frequency $\Omega_{R}(n)=\left(E_{n}^{+}-E_{n}^{-}\right) / \hbar$. It is important to remark that $E_{3}-E_{1}=2 \hbar \omega$ and so the driving field is exactly on resonance.

We now introduce a unitary operator that transforms the atomic and photonic operators in $H_{r}$ to a "dressed-operator" representation $[17,18]$. Consider the unitary operator

$$
T=\exp \left[\left(\sigma_{31} a^{2}-\sigma_{13} a^{\dagger 2}\right) \hat{\theta}_{N-2} / \sqrt{N(N-1)}\right],
$$

where

$$
\sin \hat{\theta}_{N-2}=\sqrt{N-1} / \sqrt{N\left(r^{2}+1\right)-1}
$$

and

$$
\cos \hat{\theta}_{N-2}=r \sqrt{N} / \sqrt{N\left(r^{2}+1\right)-1} .
$$

Note that $\left[N, \sigma_{31} a^{2}\right]=\left[N, \sigma_{13} a^{\dagger 2}\right]=0$. On expanding the exponential in Eq. (6), the unitary operator $T$ can be written as

$$
T=\cos \hat{\theta}_{N-2}+\left(\sigma_{31} a^{2}-\sigma_{13} a^{\dagger 2}\right) \sin \hat{\theta}_{N-2} / \sqrt{N(N-1)} .
$$

The operator $T$ is actually independent of the detuning parameter $\Delta$ owing to the independence of the dressed states on $\Delta$, which differs from the case of one-photon resonance fluorescence [17]. The dressed operator $\bar{A}$ is defined in terms of the bare operator $A$ by $\bar{A}=T A T^{\dagger}$ since $T^{-1}=T^{\dagger}$. The Hamiltonian $H_{r}$, when expressed in terms of dressed operators, becomes

$$
H_{r}=g(N)+E_{N-2}^{+} \bar{\sigma}_{11}+E_{N-2}^{-} \bar{\sigma}_{33},
$$

where $E_{N-2}^{-}$and $E_{N-2}^{+}$are operators obtained from Eq. (5) and

$$
\begin{aligned}
g(N)= & \hbar \omega+E_{0}(N)-\frac{E_{1}+E_{3}}{2}+\frac{1}{2} \Delta \\
& +\frac{1}{2} \sqrt{\Delta^{2}+4 \hbar^{2}\left[g_{1}^{2} N+g_{2}^{2}(N-1)\right]} .
\end{aligned}
$$

Note that the $\bar{\sigma}_{11}=\sum_{n=0}^{\infty}\left|\Psi_{n}^{+}\right\rangle\left\langle\Psi_{n}^{+}\left|, \bar{\sigma}_{33}=\sum_{n=0}^{\infty}\right| \Psi_{n}^{-}\right\rangle\left\langle\Psi_{n}^{-}\right|$, and the operator $N=\bar{N}$. The operator $T$ transforms the bare states into dressed states as follows, $T|3, n\rangle=\left|\Psi_{n}^{-}\right\rangle$and $T|1, n+2\rangle=\left|\Psi_{n}^{+}\right\rangle$. Accordingly, when expressed in terms of dressed operators, the effect of $T$ is to diagonalize $H_{r}$ in the dressed state representation, where the photon driving field occurs in $H_{r}$ only through the operator $N$. Note also that $N\left|\Psi_{n}^{ \pm}\right\rangle=(n+2)\left|\Psi_{n}^{ \pm}\right\rangle$. Hence the eigenvalues of $N$ would represent the strength of the pump laser.

Finally, the expression of the Hamiltonian $H$ in terms of dressed operators is completed by expressing the bare operator $\sigma_{13}$, which appears in the interacting part of the fluorescent modes in Eq. (2), in terms of dressed operator and so

$$
\sigma_{13}=\hat{s}_{1}+\hat{s}_{2}+\hat{s}_{3}+\hat{s}_{4}
$$

where

$$
\begin{gathered}
\hat{s}_{1}=\bar{\sigma}_{13} \cos \hat{\theta}_{N-4} \cos \hat{\theta}_{N-2}, \\
\hat{s}_{2}=-\bar{\sigma}_{33} \bar{a}^{2} \sin \hat{\theta}_{N-4} \cos \hat{\theta}_{N-2} / \sqrt{(N-2)(N-3)},
\end{gathered}
$$




$$
\hat{s}_{3}=-\bar{\sigma}_{31} \bar{a}^{4} \sin \hat{\theta}_{N-4} \sin \hat{\theta}_{N-2} / \sqrt{N(N-1)(N-2)(N-3)},
$$

and

$$
\hat{s}_{4}=\bar{\sigma}_{11} \bar{a}^{2} \cos \hat{\theta}_{N-4} \sin \hat{\theta}_{N-2} / \sqrt{N(N-1)} .
$$

Carets denote dressed operators; however, for conciseness, we do not use it on the operator $N$. It is important to remark that Eq. (11) is exact and does not represent an expansion for large $N$ as is necessary for one-photon resonance fluorescence [17]. Accordingly, in the two-photon resonance fluorescence case, one can treat both the weak and the strong field excitations.

The interaction term in Eq. (2), together with Eq. (11), gives rise to transitions between different eigenstates of the free part of the Hamiltonian (2) with absorption or emission of two fluorescent photons of frequency $\omega_{\mathbf{k}}$. Note that, $\bar{\sigma}_{13}\left|\Psi_{n}^{-}\right\rangle=\left|\Psi_{n-2}^{+}\right\rangle, \quad \bar{\sigma}_{33} \bar{a}^{2}\left|\Psi_{n}^{-}\right\rangle=\sqrt{N(N-1)}\left|\Psi_{n-2}^{-}\right\rangle, \quad \bar{\sigma}_{31} \bar{a}^{4}\left|\Psi_{n}^{+}\right\rangle$ $=\sqrt{N(N-1)(N+1)(N+2)}\left|\Psi_{n-2}^{-}\right\rangle, \quad$ and $\quad \bar{\sigma}_{11} \bar{a}^{2}\left|\Psi_{n}^{+}\right\rangle$ $=\sqrt{(N+1)(N+2)}\left|\Psi_{n-2}^{+}\right\rangle$.

\section{HEISENBERG EQUATIONS FOR OPERATORS}

Consider the Heisenberg equation of motion for an operator $Q$ that does not depend on the fluorescent modes, viz. $\left[Q, a_{\mathbf{k} j}\right]=0$, then

$$
\dot{Q}=-\frac{i}{\hbar}\left[Q, H_{r}\right]-\frac{i}{\hbar} \sum_{\mathbf{k} j}\left(\epsilon_{\mathbf{k} j}\left[Q, \sigma_{31}\right] a_{\mathbf{k} j}^{2}+\epsilon_{\mathbf{k} j}^{*} a_{\mathbf{k} j}^{\dagger 2}\left[Q, \sigma_{13}\right]\right) .
$$

Note that the fluorescent modes are not dressed since $\left[T, a_{\mathbf{k} j}\right]=0$.

In order to evaluate the time evolution of $Q$, we need to know the time dependence of the operators $a_{\mathbf{k} j}^{2}, a_{\mathbf{k} j}^{\dagger 2}, \sigma_{13}$, and $\sigma_{31}$. We wish to evaluate $Q$ to the first Born approximation, i.e., to second order in the coupling constant $\epsilon_{\mathrm{k} j}$ (or, to order $\left|\epsilon_{\mathbf{k} j}\right|^{2}$ ). Therefore $a_{\mathbf{k} j}^{2}$ and $a_{\mathbf{k} j}^{\dagger 2}$ will be evaluated to order $\epsilon_{\mathbf{k} j}^{*}$ and $\epsilon_{\mathrm{k} j}$, respectively, while $\sigma_{13}$ and $\sigma_{31}$ will be calculated to zeroth order in the coupling constant.

Assuming $\left\langle\left\{0_{\mathbf{q}}\right\}\left|a_{\mathbf{k} j}^{\dagger}(0) a_{\mathbf{k} j}(0)\right|\left\{0_{\mathbf{q}}\right\}\right\rangle=0$, viz., the fluorescent fields at $t=0$ are all in their respective vacuum states $\left|\left\{0_{\mathbf{q}}\right\}\right\rangle$, we have

$$
a_{\mathbf{k} j}^{2}(t)=-\frac{2 i}{\hbar} \epsilon_{\mathbf{k} j}^{*} e^{-2 i \omega_{\mathbf{k}} t} \int_{0}^{t} d t^{\prime} \sigma_{13}\left(t^{\prime}\right) e^{2 i \omega_{\mathbf{k}} t^{\prime}}
$$

where

$$
\sigma_{13}(t)=\sum_{j=1}^{4} e^{-2 i \hat{\omega}_{j}(N) t} \hat{s}_{j}(0) .
$$

The frequencies depend on $N$ and are defined by

$$
\begin{aligned}
& 2 \hbar \hat{\omega}_{1}(N)=g(N+2)-g(N)+\left(\hat{E}_{N}^{-}-\hat{E}_{N-2}^{+}\right), \\
& 2 \hbar \hat{\omega}_{2}(N)=g(N+2)-g(N)+\left(\hat{E}_{N}^{-}-\hat{E}_{N-2}^{-}\right), \\
& 2 \hbar \hat{\omega}_{3}(N)=g(N+2)-g(N)+\left(\hat{E}_{N}^{+}-\hat{E}_{N-2}^{-}\right),
\end{aligned}
$$

$$
2 \hbar \hat{\omega}_{4}(N)=g(N+2)-g(N)+\left(\hat{E}_{N}^{+}-\hat{E}_{N-2}^{+}\right) .
$$

The term $g(N+2)-g(N)$ represents a uniform shift in the frequency of the resonance fluorescence spectrum.

On integrating Eq. (14) with the aid of Eq. (15) one obtains

$$
a_{\mathbf{k} j}^{2}(t)=-\frac{i}{\hbar} \epsilon_{\mathbf{k} j}^{*} \sum_{l=1}^{4}\left[\pi \delta\left[\omega_{k}-\hat{\omega}_{l}(N)\right]+i \mathrm{P}\left(\frac{1}{\hat{\omega}_{l}(N)-\omega_{k}}\right)\right] \hat{s}_{l}(t),
$$

where $\mathrm{P}$ indicates the Cauchy principal value. The equation of motion (13) becomes, with the help of Eq. (17) and on summing over the fluorescent photon modes $\mathbf{k} j$,

$$
\begin{aligned}
\dot{Q}= & -\frac{i}{\hbar}\left[Q, H_{r}\right]-\left[Q, \sigma_{31}\right] \sum_{l=1}^{4}\left(\hat{\gamma}_{l}+i \hat{\Omega}_{l}\right) \hat{s}_{l}(t)+\sum_{l=1}^{4} \hat{s}_{l}^{\dagger}(t)\left(\hat{\gamma}_{l}\right. \\
& \left.-i \hat{\Omega}_{l}\right)\left[Q, \sigma_{13}\right],
\end{aligned}
$$

where

$$
\hat{\Omega}_{l}=\frac{1}{\hbar^{2}} \sum_{\mathbf{k} j}\left|\epsilon_{\mathbf{k} j}\right|^{2} \mathrm{P}\left(\frac{1}{\hat{\omega}_{l}(N)-\omega_{k}}\right)
$$

and

$$
\hat{\gamma}_{l}=\frac{\pi}{\hbar^{2}} \sum_{\mathbf{k} j}\left|\epsilon_{\mathbf{k} j}\right|^{2} \delta\left[\hat{\omega}_{l}(N)-\omega_{k}\right] .
$$

Next, the following approximations are made closely following the work of Persico et al. $[7,17,20]$.

(1) The terms proportional to $\hat{\Omega}_{l}$ in Eq. (18) represent part of the Lamb shifts, which contribute small changes in the frequencies of the time development of $Q$ and are thus neglected.

(2) The terms proportional to $\hat{\gamma}_{l}$ in Eq. (18) contribute to the time decay of $Q(t)$, which in the case, say, $Q(t)=\hat{s}_{l}$, determines the linewidth of the $l$ th line in the fluorescence spectrum. Accordingly, the $l$-dependence in Eq. (19) is neglected and so $\hat{\gamma}_{l}=\gamma$.

(3) Finally, for $Q(t)=\hat{s}_{l}$ in Eq. (18), the terms on the righthand side of Eq. (18) are proportional to $\hat{s}_{m}$ and so are neglected for $l \neq m$ owing to the fast oscillation of $e^{i t\left[\hat{\omega}_{l}(N)-\hat{\omega}_{m}(N)\right]}$. Note, however, that the difference $\hat{\omega}_{2}(N)$ $-\hat{\omega}_{4}(N)$ approaches zero for large $N$ and so the resulting equations for $\hat{s}_{2}$ and $\hat{s}_{4}$ are coupled whereas those for $\hat{s}_{1}$ and $\hat{s}_{3}$ are not.

The resulting equations of motions for $\hat{s}_{l}, l=1 \cdots 4$, become, with the aid of the above approximations,

$$
\begin{gathered}
\frac{d}{d t} \hat{s}_{1}=-2 i \hat{\omega}_{1}(N) \hat{s}_{1}-\hat{\Gamma}_{1}(N) \hat{s}_{1}, \\
\frac{d}{d t} \hat{s}_{2}=A(N) \hat{s}_{2}+B(N) \hat{s}_{4},
\end{gathered}
$$




$$
\frac{d}{d t} \hat{s}_{3}=-2 i \hat{\omega}_{3}(N) \hat{s}_{3}-\hat{\Gamma}_{3}(N) \hat{s}_{3}
$$

and

$$
\frac{d}{d t} \hat{s}_{4}=D(N) \hat{s}_{4}+C(N) \hat{s}_{2}
$$

where

$$
\begin{gathered}
\hat{\Gamma}_{1}(N)=\gamma \cos ^{2} \hat{\theta}_{N}+\gamma \sin ^{2} \hat{\theta}_{N-2}+2 \gamma \cos ^{2} \hat{\theta}_{N-4} \sin ^{2} \hat{\theta}_{N-2} \\
\hat{\Gamma}_{3}(N)=\gamma \sin ^{2} \hat{\theta}_{N}+\gamma \cos ^{2} \hat{\theta}_{N-2}+2 \gamma \sin ^{2} \hat{\theta}_{N-4} \cos ^{2} \hat{\theta}_{N-2} \\
A(N)=-2 i \hat{\omega}_{2}(N)-\gamma \cos ^{2} \hat{\theta}_{N}-\gamma \cos ^{2} \hat{\theta}_{N-2} \cos \left(2 \hat{\theta}_{N-4}\right) \\
B(N)=-2 \gamma \sin ^{2} \hat{\theta}_{N-4} \sin ^{2} \hat{\theta}_{N-2} \\
C(N)=-2 \gamma \cos ^{2} \hat{\theta}_{N-4} \cos ^{2} \hat{\theta}_{N-2}
\end{gathered}
$$

and

$$
D(N)=-2 i \hat{\omega}_{4}(N)-\gamma \sin ^{2} \hat{\theta}_{N}+\gamma \sin ^{2} \hat{\theta}_{N-2} \cos \left(2 \hat{\theta}_{N-4}\right) .
$$

Note that the equations of motion for $\hat{s}_{2}$ and $\hat{s}_{4}$ are coupled owing to $\hat{\omega}_{4}(N)-\hat{\omega}_{2}(N) \simeq \sqrt{g_{1}^{2}+g_{2}^{2}} / 2 \sqrt{N}$ for large values of the eigenvalues of the operator $N$. It is shown below that the terms associated with $\hat{s}_{2}$ and $\hat{s}_{4}$ give rise to the elastic scattering term in the resonance fluorescence spectrum.

\section{SOLUTIONS OF HEISENBERG EQUATIONS}

The solutions to Eq. (20) follow by direct integration and are

$$
\begin{gathered}
\hat{s}_{1}\left(t^{\prime}\right)=e^{-\left[2 i \hat{\omega}_{1}(N)+\hat{\Gamma}_{1}(N)\right]\left(t^{\prime}-t^{\prime \prime}\right)} \hat{s}_{1}\left(t^{\prime \prime}\right), \\
\hat{s}_{3}\left(t^{\prime}\right)=e^{-\left[2 i \hat{\omega}_{3}(N)+\hat{\Gamma}_{3}(N)\right]\left(t^{\prime}-t^{\prime \prime}\right)} \hat{s}_{3}\left(t^{\prime \prime}\right), \\
\hat{s}_{2}\left(t^{\prime}\right)=\frac{e^{\left[A(N)+\alpha_{+}(N)\right]\left(t^{\prime}-t^{\prime \prime}\right)}}{\alpha_{-}(N)-\alpha_{+}(N)}\left\{\alpha_{-}(N) \hat{s}_{2}\left(t^{\prime \prime}\right)-B(N) \hat{s}_{4}\left(t^{\prime \prime}\right)\right\} \\
+\frac{e^{\left[A(N)+\alpha_{-}(N)\right]\left(t^{\prime}-t^{\prime \prime}\right)}}{\alpha_{-}(N)-\alpha_{+}(N)}\left\{B(N) \hat{s}_{4}\left(t^{\prime \prime}\right)-\alpha_{+}(N) \hat{s}_{2}\left(t^{\prime \prime}\right)\right\},
\end{gathered}
$$

and

$$
\begin{aligned}
\hat{s}_{4}\left(t^{\prime}\right)= & \frac{e^{\left[A(N)+\alpha_{+}(N)\right]\left(t^{\prime}-t^{\prime \prime}\right)}}{\alpha_{-}(N)-\alpha_{+}(N)}\left\{-C(N) \hat{s}_{2}\left(t^{\prime \prime}\right)-\alpha_{+}(N) \hat{s}_{4}\left(t^{\prime \prime}\right)\right\} \\
& +\frac{e^{\left[A(N)+\alpha_{-}(N)\right]\left(t^{\prime}-t^{\prime \prime}\right)}}{\alpha_{-}(N)-\alpha_{+}(N)}\left\{C(N) \hat{s}_{2}\left(t^{\prime \prime}\right)+\alpha_{-}(N) \hat{s}_{4}\left(t^{\prime \prime}\right)\right\},
\end{aligned}
$$

where

$$
\alpha_{ \pm}(N)=\frac{1}{2}\left\{D(N)-A(N) \pm \sqrt{[A(N)-D(N)]^{2}+4 B(N) C(N)}\right\} .
$$

\section{FLUORESCENCE SPECTRUM}

In one-photon resonance fluorescence, the excited atom spontaneously emits light of the same frequency as that of the driving field when the excitation is weak [21]. In strong fields, however, the fluorescence spectrum has three-peak sideband structure [1]. In this paper, we investigate the spectrum of two-photon resonance fluorescence for arbitrary initial states of the radiation field and atoms in the atomic beam. Quantitative analysis of the spectrum starts with an evaluation of the average number of spontaneously emitted photons by the dressed atom in the mode $(\mathbf{k} j)$ [11]. The average $\left\langle a_{\mathbf{k} l}^{\dagger}(t) a_{\mathbf{k} l}(t)\right\rangle$ is taken over initial states that are devoid of fluorescent photons and over arbitrary initial states of the atoms in the atomic beam and the photon driving field, which may be a laser.

The equation for the fluorescent photon number operator in the $(\mathbf{k} l)$ mode is given by

$$
i \hbar \frac{d}{d t}\left(a_{\mathbf{k} l}^{\dagger} a_{\mathbf{k} l}\right)=2\left(\epsilon_{\mathbf{k} j}^{*} a_{\mathbf{k} j}^{\dagger 2} \sigma_{13}-\epsilon_{\mathbf{k} j} a_{\mathbf{k} j}^{2} \sigma_{31}\right)
$$

Equation (24) can be integrated to give the average number of photons at time $t$,

$$
\left\langle a_{\mathbf{k} l}^{\dagger}(t) a_{\mathbf{k} l}(t)\right\rangle=\frac{2 i}{\hbar} \epsilon_{\mathbf{k} j}\left\langle\int_{0}^{t} d t^{\prime} a_{\mathbf{k} j}^{2}\left(t^{\prime}\right) \sigma_{31}\left(t^{\prime}\right)\right\rangle+\text { H.c. }
$$

Note that the average on the right-hand side of Eq. (25) is over the states of initial atoms, and pump and fluorescent photon fields. Using the Born approximation for $a_{\mathbf{k} j}^{2}\left(t^{\prime}\right)$ and $a_{\mathbf{k} j}^{\dagger 2}\left(t^{\prime}\right)$ and since there are no fluorescent photons at $t=0$, we obtain

$$
\begin{aligned}
\left\langle a_{\mathbf{k} l}^{\dagger}(t) a_{\mathbf{k} l}(t)\right\rangle= & \frac{8}{\hbar^{2}}\left|\epsilon_{\mathbf{k} l}\right|^{2} \operatorname{Re} \int_{0}^{t} d t^{\prime} \int_{0}^{t^{\prime}} d t^{\prime \prime} e^{-2 i \omega_{\mathbf{k}}\left(t^{\prime}-t^{\prime \prime}\right)} \\
& \times\left\langle\sigma_{31}\left(t^{\prime}\right) \sigma_{13}\left(t^{\prime \prime}\right)\right\rangle,
\end{aligned}
$$

where the average of the correlation function in Eq. (26) is over the initial states of the atoms and photon driving field, which may be represented in terms of the eigenstates (3) of the Hamiltonian $H_{r}$ given by Eq. (1), and Re denotes the real part. The nonzero terms in the correlation function in Eq. (26) are $\left\langle\sigma_{31}\left(t^{\prime}\right) \sigma_{13}\left(t^{\prime \prime}\right)\right\rangle=\sum_{j=1}^{4}\left\langle s_{j}^{\dagger}\left(t^{\prime}\right) s_{j}\left(t^{\prime \prime}\right)\right\rangle$. Using the results of Eq. (22), $\left\langle a_{\mathbf{k} l}^{\dagger}(t) a_{\mathbf{k} l}(t)\right\rangle$ can be evaluated by performing the time integrations and keeping the $t$-dependent terms, which are the leading terms for large values of $t$, and so one obtains for the rate of production of fluorescent photons 


$$
\begin{aligned}
\frac{1}{t}\left\langle a_{\mathbf{k} l}^{\dagger}(t) a_{\mathbf{k} l}(t)\right\rangle= & \frac{8}{\hbar^{2}}\left|\epsilon_{\mathbf{k} l}\right|^{2}\left[\left\langle\frac{\hat{\Gamma}_{1}(N-2) \cos ^{2} \hat{\theta}_{N-4} \cos ^{2} \hat{\theta}_{N-2}}{\left[2 \omega_{\mathbf{k}}-2 \hat{\omega}_{1}(N-2)\right]^{2}+\hat{\Gamma}_{1}^{2}(N-2)} \bar{\sigma}_{33}\right\rangle+\left\langle\frac{\hat{\Gamma}_{3}(N-2) \sin ^{2} \hat{\theta}_{N-4} \sin ^{2} \hat{\theta}_{N-2}}{\left[2 \omega_{\mathbf{k}}-2 \hat{\omega}_{3}(N-2)\right]^{2}+\hat{\Gamma}_{3}^{2}(N-2)} \bar{\sigma}_{11}\right\rangle\right. \\
& +\left\langle\frac{\hat{\Gamma}_{e}(N-2) \operatorname{Re}\left[\hat{M}_{e}(N-2)\right]+2\left[\omega_{\mathbf{k}}-\hat{\omega}_{e}(N-2)\right] \operatorname{Im}\left[\hat{M}_{e}(N-2)\right]}{\left[2 \omega_{\mathbf{k}}-2 \hat{\omega}_{e}(N-2)\right]^{2}+\hat{\Gamma}_{e}^{2}(N-2)}\right\rangle \\
& \left.+\left\langle\frac{\hat{\Gamma}_{i} \operatorname{Re}\left[\hat{M}_{i}(N-2)\right]+2\left[\omega_{\mathbf{k}}-\hat{\omega}_{i}(N-2)\right] \operatorname{Im}\left[\hat{M}_{i}(N-2)\right]}{\left[2 \omega_{\mathbf{k}}-2 \hat{\omega}_{i}(N-2)\right]^{2}+\hat{\Gamma}_{i}^{2}(N-2)}\right\rangle\right],
\end{aligned}
$$

with

$$
\begin{aligned}
\hat{M}_{e}(N)= & \frac{1}{\left[\alpha_{-}^{*}(N)-\alpha_{+}^{*}(N)\right]}\left[\left[\alpha_{-}^{*}(N)\right.\right. \\
& -C(N)] \sin ^{2} \hat{\theta}_{N-2} \cos ^{2} \hat{\theta}_{N} \bar{\sigma}_{33}-[B(N) \\
& \left.\left.+\alpha_{+}^{*}(N)\right] \cos ^{2} \hat{\theta}_{N-2} \sin ^{2} \hat{\theta}_{N} \bar{\sigma}_{11}\right], \\
\hat{M}_{i}(N)= & \frac{1}{\left[\alpha_{-}^{*}(N)-\alpha_{+}^{*}(N)\right]}[[C(N) \\
& \left.-\alpha_{+}^{*}(N)\right] \sin ^{2} \hat{\theta}_{N-2} \cos ^{2} \hat{\theta}_{N} \bar{\sigma}_{33}+[B(N) \\
& \left.\left.+\alpha_{-}^{*}(N)\right] \cos ^{2} \hat{\theta}_{N-2} \sin ^{2} \hat{\theta}_{N} \bar{\sigma}_{11}\right],
\end{aligned}
$$

where $\hat{\omega}_{e}(N), \hat{\omega}_{i}(N), \hat{\Gamma}_{e}(N)$, and $\hat{\Gamma}_{i}(N)$ are defined by

$$
\hat{\omega}_{e}(N)=\frac{1}{2} \operatorname{Im}\left[A^{*}(N)+\alpha_{+}^{*}(N)\right], \quad \Gamma_{e}=-\operatorname{Re}\left[A^{*}(N)+\alpha_{+}^{*}(N)\right],
$$

and

$$
\hat{\omega}_{i}(N)=\frac{1}{2} \operatorname{Im}\left[A^{*}(N)+\alpha_{-}^{*}(N)\right], \quad \Gamma_{i}=-\operatorname{Re}\left[A^{*}(N)+\alpha_{-}^{*}(N)\right],
$$

where Re and Im are the real and imaginary parts, respectively. Note that in the expectation values on the right-hand side of Eq. (27), the state $\left|\Psi_{n}^{-}\right\rangle$contributes in the first term, the state $\left|\Psi_{n}^{+}\right\rangle$contributes in the second term, and both states contribute in the third and fourth terms.

In a typical resonance fluorescence experiment before the atoms enter the region of the pump field, the atom-field beams system is in the disentangled state $\rho_{i n}=\rho_{R} \otimes \rho_{A}$. An arbitrary atomic state is represented by $\rho_{A}=\sum_{i, j=1,3} A_{i, j}|i\rangle\langle j|$ with $\operatorname{tr} \rho_{A}=1$. The arbitrary pump field is represented by $\rho_{R}$ $=\sum_{n, n^{\prime}=0}^{\infty} R_{n, n^{\prime}}|n\rangle\left\langle n^{\prime}\right|$ with tr $\rho_{R}=1$. Therefore, with the aid of Eq. (3), one has for an arbitrary function of the operator $N$,

$$
\begin{aligned}
\left\langle f(N) \bar{\sigma}_{11}\right\rangle= & \sum_{n=0}^{\infty} R_{n, n} A_{1,1} \cos ^{2} \theta_{n-2} f(n) \\
& +\sum_{n=0}^{\infty} R_{n, n-2} A_{1,3} \sin \theta_{n-2} \cos \theta_{n-2} f(n) \\
& +\sum_{n=0}^{\infty} R_{n, n+2} A_{3,1} \sin \theta_{n} \cos \theta_{n} f(n+2) \\
& +\sum_{n=0}^{\infty} R_{n, n} A_{3,3} \sin ^{2} \theta_{n} f(n+2),
\end{aligned}
$$

and

$$
\begin{aligned}
\left\langle f(N) \bar{\sigma}_{33}\right\rangle= & \sum_{n=0}^{\infty} R_{n, n} A_{1,1} \sin ^{2} \theta_{n-2} f(n) \\
& -\sum_{n=0}^{\infty} R_{n, n-2} A_{1,3} \sin \theta_{n-2} \cos \theta_{n-2} f(n) \\
& -\sum_{n=0}^{\infty} R_{n, n+2} A_{3,1} \sin \theta_{n} \cos \theta_{n} f(n+2) \\
& +\sum_{n=0}^{\infty} R_{n, n} A_{3,3} \cos ^{2} \theta_{n} f(n+2),
\end{aligned}
$$

which gives $\langle f(N)\rangle=\sum_{n=0}^{\infty} R_{n, n} A_{1,1} f(n)+\sum_{n=0}^{\infty} R_{n, n} A_{3,3} f(n+2)$. Note that for strong driving fields, $f(n+2) \simeq f(n)$ and so $\langle f(N)\rangle \simeq \sum_{n=0}^{\infty} f(n) R_{n, n}=\operatorname{tr}\left[f\left(a^{\dagger} a\right) \rho_{R}\right]$, which does not depend on the initial state of the atoms as they enter the interaction region comprising the atomic and laser beams. In particular, if the driving field is a strong laser, described by a coherent state $|\xi\rangle$ with $R_{n, n^{\prime}}=e^{-|\xi|^{2}} \xi^{n} \xi^{*^{\prime}} / \sqrt{n ! n^{\prime} !}$ peaked at $n=|\xi|^{2}$ where the width becomes arbitrarily narrow for $|\xi|^{2} \gg 1$, one has that $\langle f(N)\rangle=f\left(\left\langle a^{\dagger} a\right\rangle\right)$, where $\left\langle a^{\dagger} a\right\rangle=|\xi|^{2}$ is the average number of photons in the laser field, which is proportional to the intensity of the laser beam. One should remark that the $c$-number description of the driving field is strictly equivalent to the quantum description provided the state of the field is a coherent state [22,23]. However, our result [Eq. (27)] applies equally well to arbitrary quantum states of the driving field. 
The fluorescent spectrum given by Eq. (27) is valid for arbitrary values of the eigenvalues of the excitation operator $N$. The spectrum shows four peaks, two Lorentzians located, respectively, at $2 \omega_{\mathbf{k}}=2 \hat{\omega}_{1}(N-2), 2 \hat{\omega}_{3}(N-2)$ and two nonLorentzian located, respectively, at $2 \omega_{\mathbf{k}}=2 \hat{\omega}_{e}(N-2), 2 \hat{\omega}_{i}(N$ $-2)$. The Lorentzian peak at $\hat{\omega}_{1}(n)$ arises from the spontaneous emission from the dressed atom $\left|\Psi_{n}^{-}\right\rangle \rightarrow\left|\Psi_{n-2}^{+}\right\rangle$for $E_{n}^{-}$ $>E_{n-2}^{+}$or $\left|\Psi_{n-2}^{+}\right\rangle \rightarrow\left|\Psi_{n}^{-}\right\rangle$for $E_{n-2}^{+}>E_{n}^{-}$and at $\hat{\omega}_{3}(n)$ from the spontaneous emission $\left|\Psi_{n}^{+}\right\rangle \rightarrow\left|\Psi_{n-2}^{-}\right\rangle$, with widths given by Eq. (21). The locations of the non-Lorentzian peaks are given by

$$
\begin{aligned}
& \hat{\omega}_{e}(N)=\frac{1}{2}\left[\hat{\omega}_{2}(N)+\hat{\omega}_{4}(N)\right]-\frac{1}{4 \sqrt{2}}\left[\left(a^{2}+b^{2}\right)^{1 / 2}-a\right]^{1 / 2}, \\
& \hat{\omega}_{i}(N)=\frac{1}{2}\left[\hat{\omega}_{2}(N)+\hat{\omega}_{4}(N)\right]+\frac{1}{4 \sqrt{2}}\left[\left(a^{2}+b^{2}\right)^{1 / 2}-a\right]^{1 / 2},
\end{aligned}
$$

where

$$
\begin{aligned}
a= & -4\left[\hat{\omega}_{4}(N)-\hat{\omega}_{2}(N)\right]^{2}+\gamma^{2}\left[\cos 2 \hat{\theta}_{N}+\cos 2 \hat{\theta}_{N-4}\right]^{2} \\
& +\gamma^{2} \sin ^{2} 2 \hat{\theta}_{N-4} \sin ^{2} 2 \hat{\theta}_{N-2}, \\
b & =-4 \gamma\left[\hat{\omega}_{4}(N)-\hat{\omega}_{2}(N)\right]\left[\cos 2 \hat{\theta}_{N}+\cos 2 \hat{\theta}_{N-4}\right] .
\end{aligned}
$$

The corresponding widths $\hat{\Gamma}_{e}$ and $\hat{\Gamma}_{i}$ are given by

$$
\begin{aligned}
& \hat{\Gamma}_{e}=\frac{\gamma}{2}+\frac{\gamma}{2} \cos 2 \hat{\theta}_{N-2} \cos 2 \hat{\theta}_{N-4}-\frac{1}{2 \sqrt{2}}\left[\left(a^{2}+b^{2}\right)^{1 / 2}+a\right]^{1 / 2}, \\
& \hat{\Gamma}_{i}=\frac{\gamma}{2}+\frac{\gamma}{2} \cos 2 \hat{\theta}_{N-2} \cos 2 \hat{\theta}_{N-4}+\frac{1}{2 \sqrt{2}}\left[\left(a^{2}+b^{2}\right)^{1 / 2}+a\right]^{1 / 2} .
\end{aligned}
$$

It is to be noted that the non-Lorentzian peaks of the spectrum at $\hat{\omega}_{e}(N)$ and $\hat{\omega}_{i}(N)$ are functions both of $\left[\hat{\omega}_{2}(N)\right.$ $\left.+\hat{\omega}_{4}(N)\right]$ and $\left[\hat{\omega}_{2}(N)-\hat{\omega}_{4}(N)\right]$, whereas $\hat{\Gamma}_{e}$ and $\hat{\Gamma}_{i}$ are only functions of $\left[\hat{\omega}_{2}(N)-\hat{\omega}_{4}(N)\right]$.

\section{A. Large $N$ limit}

The resonance fluorescence spectrum (27) has explicit dependence on $N$. The spectrum (27) simplifies considerably for values of $N$ when one can approximate $N \pm j \approx N$, where $j$ is a small integer, say, $j=2,4$. In addition, the value of $N$ can be compared to the detuning parameter $\Delta$ as it occurs, say, in $E_{n}^{+}$in Eq. (5). In the latter case, one can introduce the notions of the limit of small or large detuning.

Let us consider first the case when one sets $N \pm j \approx N$. Then the resonance fluorescence spectrum (27) becomes

$$
\begin{aligned}
\frac{1}{t}\left\langle a_{\mathbf{k} l}^{\dagger}(t) a_{\mathbf{k} l}(t)\right\rangle= & \frac{8}{\hbar^{2}}\left|\epsilon_{\mathbf{k} l}\right|^{2}\left[\left\langle\frac{\Gamma \cos ^{4} \theta}{\left[2 \omega_{\mathbf{k}}-2 \hat{\omega}_{-}(N)\right]^{2}+\Gamma^{2}} \bar{\sigma}_{33}\right\rangle\right. \\
& +\left\langle\frac{\Gamma \sin ^{4} \theta}{\left[2 \omega_{\mathbf{k}}-2 \hat{\omega}_{+}(N)\right]^{2}+\Gamma^{2}} \bar{\sigma}_{11}\right\rangle \\
& \left.+\frac{\Gamma_{i}}{\left(2 \omega_{\mathbf{k}}-2 \omega\right)^{2}+\Gamma_{i}^{2}}\left\langle\hat{m}_{i}\right\rangle\right]+\frac{4 \pi}{\hbar^{2}}\left|\epsilon_{\mathbf{k} l}\right|^{2} \delta\left(2 \omega_{\mathbf{k}}\right. \\
& -2 \omega)\left\langle\hat{m}_{e}\right\rangle,
\end{aligned}
$$

where $\cos \theta=r / \sqrt{r^{2}+1}$,

$$
\begin{gathered}
2 \hbar \hat{\omega}_{ \pm}(N)=2 \hbar \omega \mp \Delta / 2 \pm \frac{1}{2} \sqrt{\Delta^{2}+4 \hbar^{2}\left(g_{1}^{2}+g_{2}^{2}\right) N}, \\
\Gamma=\gamma+2 \gamma r^{2} /\left(1+r^{2}\right)^{2}, \quad \Gamma_{i}=2 \gamma\left(1+r^{4}\right) /\left(1+r^{2}\right)^{2}, \\
\hat{m}_{e}=\frac{r^{2}\left(1-r^{2}\right)}{\left(1+r^{2}\right)\left(1+r^{4}\right)}\left[\bar{\sigma}_{33}-\bar{\sigma}_{11}\right], \quad \hat{m}_{i} \\
=\frac{2 r^{2}}{\left(1+r^{4}\right)\left(1+r^{2}\right)^{2}}\left[r^{4} \bar{\sigma}_{33}+\bar{\sigma}_{11}\right] .
\end{gathered}
$$

The terms in the square brackets in Eq. (33) represent the inelastic spectrum for two-photon resonance florescence, which has the Mollow three-peaked structure of one-photon resonance fluorescence [1], with Lorentzians peaked at $2 \hat{\omega}_{-}(N), 2 \hat{\omega}_{+}(N)$, and $2 \omega$, respectively. As can be seen, the sidebands depend on the intensity of the incident laser radiation and are symmetrically spaced vis-à-vis the central peak as in the one-photon case. However, that is not so for finite $N$ when the spectrum is given by the more general expression (27).

The last term in Eq. (33) corresponds to the elastic or Rayleigh component peaked at twice the pump mode frequency $\omega$. It is important to remark that the elastic scattering component is finite for large $N$ provided $r \neq 1$. This is contrary to the one-photon resonance florescence case where the elastic term is of $O(1 / N)$ as $N \rightarrow \infty$ [17].

\section{B. Large and small detuning}

In analyzing the fluorescence spectrum given by Eq. (33), one can introduce limits of large and small detuning, i.e., $4 \hbar^{2}\left(g_{1}^{2}+g_{2}^{2}\right) N \ll \Delta^{2}$ and $4 \hbar^{2}\left(g_{1}^{2}+g_{2}^{2}\right) N \gg \Delta^{2}$. In the large detuning limit, the frequency shift of the sidebands from the central peak goes like $\hbar\left(g_{1}^{2}+g_{2}^{2}\right) N / \Delta$ and $\omega_{ \pm} \simeq \omega$. However, for small detuning the frequency shifts of the sidebands from the central peak goes like $\left[\sqrt{\left(g_{1}^{2}+g_{2}^{2}\right) N}-\Delta / 2 \hbar\right]$, which is proportional to the square root of the intensity of the pump field. The case of $\Delta=0$, of course, is also possible for particular three-level atoms/molecules.

\section{Fluorescence of ground-state atoms}

The spectrum given by Eq. (33) holds for arbitrary initial pump photonic and atomic states. If the atoms enter the region of the pump field in the ground state, as will be the situation in fluorescence experiments, the spectrum simpli- 
fies considerably. We now analyze the case where the atoms in the beam are initially in the ground state $|1\rangle$ and the pump field is now assumed to be in a coherent state $|\xi\rangle$, such as might be produced by an ideal intense laser and so $\rho_{\text {in }}$ $=|1\rangle\langle 1|\otimes| \xi\rangle\langle\xi|$, then Eq. (33) becomes

$$
\begin{aligned}
\frac{1}{t}\left\langle a_{\mathbf{k} l}^{\dagger}(t) a_{\mathbf{k} l}(t)\right\rangle= & \frac{8}{\hbar^{2}}\left|\epsilon_{\mathbf{k} l}\right|^{2}\left[\frac{r^{4}}{\left(r^{2}+1\right)^{3}} \frac{\Gamma}{\left[2 \omega_{\mathbf{k}}-2 \hat{\omega}_{-}\left(|\xi|^{2}\right)\right]^{2}+\Gamma^{2}}\right. \\
& +\frac{r^{2}}{\left(r^{2}+1\right)^{3}} \frac{\Gamma}{\left[2 \omega_{\mathbf{k}}-2 \hat{\omega}_{+}\left(|\xi|^{2}\right)\right]^{2}+\Gamma^{2}} \\
& \left.+\frac{2 r^{4}}{\left(1+r^{4}\right)\left(1+r^{2}\right)^{2}} \frac{\Gamma_{i}}{\left(2 \omega_{\mathbf{k}}-2 \omega\right)^{2}+\Gamma_{i}^{2}}\right] \\
& +\frac{4 \pi}{\hbar^{2}}\left|\epsilon_{\mathbf{k} l}\right|^{2} \frac{r^{2}\left(1-r^{2}\right)^{2}}{\left(1+r^{4}\right)\left(1+r^{2}\right)^{2}} \delta\left(2 \omega_{\mathbf{k}}-2 \omega\right) .
\end{aligned}
$$

The ratios of intensities at the Lorentzian peaks of the spectrum as given by Eq. (35) are

$$
\begin{gathered}
\frac{I_{\omega_{-}}}{I_{\omega}}=\frac{\left(1+r^{4}\right)^{2}}{\left(1+r^{2}\right)\left(1+4 r^{2}+r^{4}\right)}, \\
\frac{I_{\omega_{+}}}{I_{\omega}}=\frac{\left(1+r^{4}\right)^{2}}{r^{2}\left(1+r^{2}\right)\left(1+4 r^{2}+r^{4}\right)} .
\end{gathered}
$$

For $r=1, I_{\omega_{-}} / I_{\omega}=I_{\omega_{+}} / I_{\omega}=1 / 3$. However, for $r \neq 1$, we obtain different intensity ratios depending upon whether $r>1$ or $r$ $<1$. The ratio of the widths is

$$
\frac{\Gamma}{\Gamma_{i}}=\frac{\left(1+4 r^{2}+r^{4}\right)}{2\left(1+r^{4}\right)} .
$$

For $r=1$, we obtain $\Gamma / \Gamma_{i}=3 / 2$.

The spectrum $\frac{\hbar^{2} \gamma}{\left.8 t \epsilon_{\mathbf{k}}\right|^{2}}\left\langle a_{\mathbf{k} l}^{\dagger}(t) a_{\mathbf{k} l}(t)\right\rangle$ is actually a function of $r=g_{1} / g_{2}$ and the Rabi frequency for two-photon resonance fluorescence divided by $\gamma$, that is, $\Omega_{R}\left(|\xi|^{2}\right) / \gamma$, where $\hbar \Omega_{R}\left(|\xi|^{2}\right)=-\Delta / 2+\sqrt{(\Delta / 2)^{2}+\hbar^{2}\left(g_{1}^{2}+g_{2}^{2}\right)|\xi|^{2}}$. To illustrate the dependence of the fluorescence spectrum on the coupling constant ratio $r$, we plot the inelastic part of the spectrum for $r=0.5,1.0$, and 1.5, in Figs. 1-3. We choose values of $\Delta$ $=0, g_{2}=7 \times 10^{5} \mathrm{~s}^{-1}[16], \gamma=\frac{1}{3} \times 10^{-3} \mathrm{~s}^{-1}$, and $|\xi|^{2}=200$. The parameter $\Omega_{R}\left(|\xi|^{2}\right) / \gamma$ was scaled by a factor of $10^{-3}$.

\section{COMPARISON WITH OTHER THEORIES}

Two-photon resonance fluorescence calculated in Refs. [12-14] follows the Green's function method. In Ref. [14], Basu et al. assumed a phenomenological two-photon Hamiltonian. However, the coupling constant is arbitrary. While this comes close to the two photon interaction Hamiltonian (1) obtained previously by us [16], our result obtained by a unitary transformation is exact, and the coupling constant is not arbitrary. As stated in Ref. [14], the results are valid for high pump field intensity. The spectrum shows three Lorentzian lines: one at twice the pump frequency and two symmetrically located sidebands, whose frequency shift from the

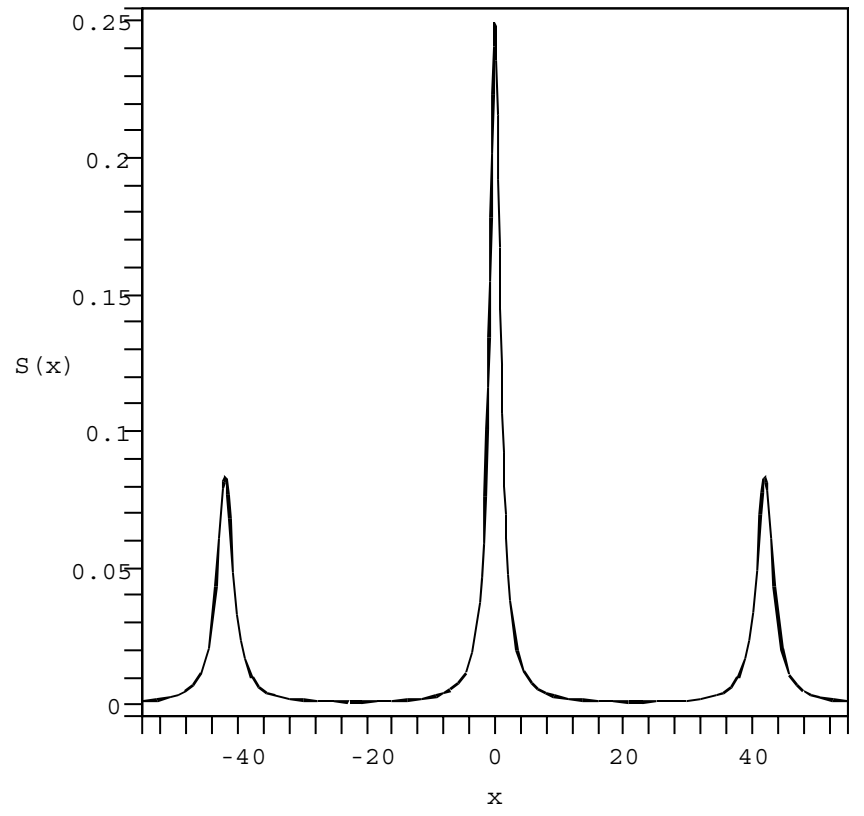

FIG. 1. Two-photon resonant-fluorescence spectrum $S(x)$ vs $x$, where $S(x)=\hbar^{2} \gamma / 8 t\left|\epsilon_{\mathbf{k} l}\right|^{2}\left\langle a_{\mathbf{k} l}^{\dagger}(t) a_{\mathbf{k} l}(t)\right\rangle, \quad x=2\left(\omega_{\mathbf{k}}-\omega\right) / \gamma$, and the $\delta$-function in Eq. (35) is not included. Choose $\Delta=0, g_{1}=g_{2}=7$ $\times 10^{5} \mathrm{~s}^{-1}, \gamma=\frac{1}{3} \times 10^{3} \mathrm{~s}^{-1}$, and $|\xi|^{2}=200$ [16]. The positions of the sidebands have been rescaled by the factor of $10^{-3}$, i.e., the actual positions of the sidebands are at \pm 42000 .

main peak depends on the pump field intensity. However, the spectrum is otherwise identical to that found by Mollow [1] for the one-photon case but without the elastic peak. On the other hand, Mavroyannis et al. [12] found the spectrum to have a four-peak structure, an intense peak at twice the pump frequency, two sidebands, and a weak peak at the pump frequency. The widths of the three peaks are equal and one-half that of the one-photon peak.

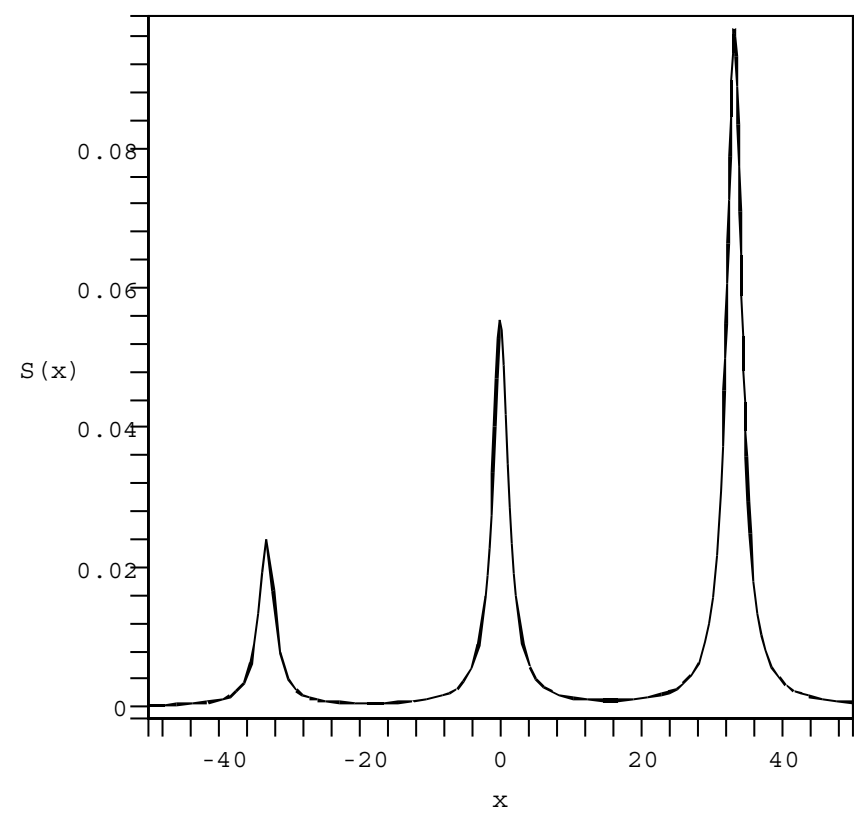

FIG. 2. Same as Fig. 1 but with $g_{2}=7 \times 10^{5} \mathrm{~s}^{-1}$ and $r=g_{1} / g_{2}$ $=0.5$. The actual positions of the sidebands are at \pm 32000 . 


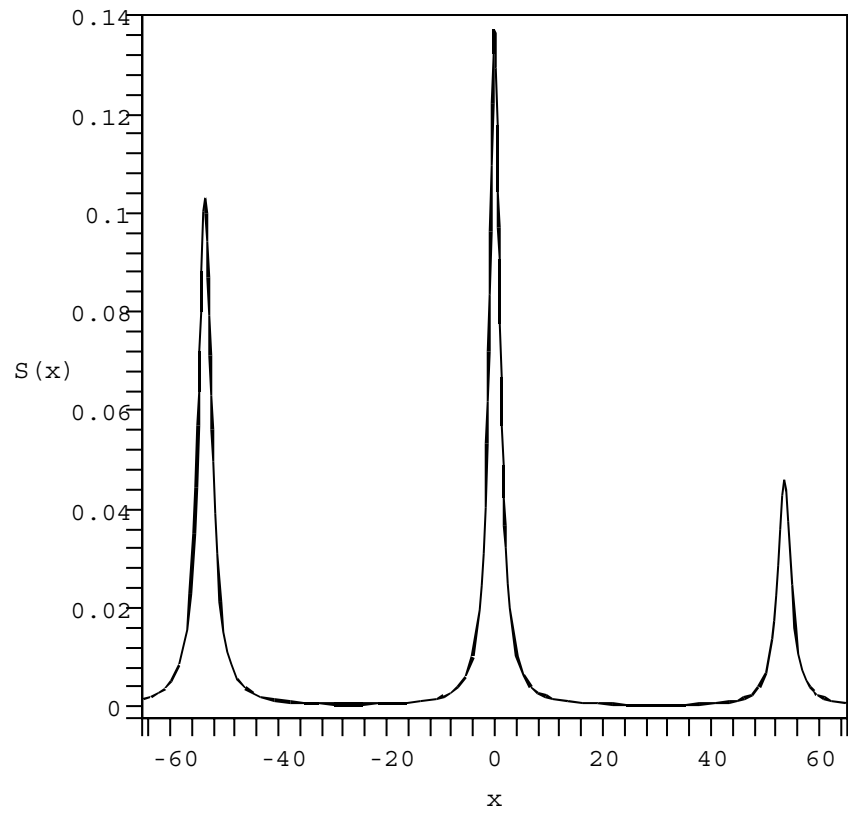

FIG. 3. Same as Fig. 1 but with $g_{2}=7 \times 10^{5} \mathrm{~s}^{-1}$ and $r=g_{1} / g_{2}$ $=1.5$. The actual positions of the sidebands are at \pm 54000 .

In Ref. [13], Cheung takes into account energy nonconserving terms in the Hamiltonian. The fluorescence spectrum shows four Lorentzians. The results are similar to those of Ref. [12], except that the peaks appear with different heights. In Ref. [11], Holm and Sargent consider the rate of production of fluorescent photons, as is done by us, to calculate the spectrum of fluorescence. However, the pump field is taken to be classical. In this theory, the two fluorescent photons are allowed to have different frequencies. The spectrum is fairly complicated. Interestingly, an elastic component, the Rayleigh peak, is also found in the spectrum, similar to the result obtained in this paper for large $N$. Note that none of the results of Refs. [12-15] show an elastic peak. The work of Ref. [15] is based on adapting the Heitler-Ma nonperturbative method for single-photon transition to the case of two- photon resonance fluorescence. An effective interaction Hamiltonian is introduced but their results are rather puzzling since the spectrum is effectively indistinguishable for the cases of weak or strong excitation fields.

The fluorescence spectrum derived by us, using the dressed-atom formalism, is valid for arbitrary values of the excitation number $N$. The most general spectrum given by Eq. (27) has four peaks, two Lorentzian and two nonLorentzian lines. The spectrum is quite different from those found in pervious works. The location as well as the widths of the peaks are functions of $N$. In the large $N$ limit, the spectrum shows three Lorentzians and an elastic scattering or Raleigh component.

The fluorescence spectrum for large $N$, Eqs. (33) and (35), show a sensitive dependence on $r$, the ratio of the coupling constants of the $1 \rightarrow 2$ and $2 \rightarrow 3$ atomic transitions. The positions as well as the widths are sensitive functions of $r$. This is a result not found in any previous works on two-photon fluorescence. For $r=1$, the elastic scattering term vanishes. The ratio of widths of the sidebands to the width of the central peak in this case is $3 / 2$, whereas the ratio of the intensity of the central peak to the intensities of the sidebands is 3 .

\section{SUMMARY AND CONCLUSIONS}

The theory of two-photon fluorescence presented here is useful in the context of recent developments in two-photon fluorescence microscopy as diagnostic and imaging tools in medicine and biology. We have used the dressed-atom representation that allows evaluation of the resonance fluorescence spectrum in high pump field excitations. The most general spectrum for arbitrary excitations exhibits four peaks, two Lorentzian and two non-Lorentzian terms. Such a spectrum has not been seen in previous theories of twophoton fluorescence. In the large excitation limit, the spectrum shows three Lorentzian peaks and an elastic scattering component. The spectrum shows a sensitive dependence on the ratio of the coupling constants of the three-level atom.
[1] B. R. Mollow, Phys. Rev. 188, 1969 (1969).

[2] F. Schuda, C. R. Stroud, and M. Hercher, J. Phys. B 7, L 198 (1974).

[3] W. Denk, J. Strickler, and W. Webb, Science 248, 76 (1990).

[4] P. T. C. So, C. Y. Dong, B. R. Masters, and K. M. Berland, Annu. Rev. Biomed. Eng. 2, 399 (2000).

[5] D. Bird and M. Gu, Opt. Lett. 28, 1552 (2003); A. K. Dunn, V. P. Wallace, M. Coleno, M. W. Berns, and B. J. Tromberg, Appl. Opt. 39, 1194 (2000); T. French, P. T. C. So, D. J. Weaver, Jr., T. Coelho-Sampaio, E. Gratton, E. W. Voss, Jr., and J. Carrero, J. Microsc. 185, 339 (1997).

[6] R. A. Farrer, M. J. R. Previte, C. E. Olson, L. A. Peyser, J. T. Fourkas, and P. T. C. So, Opt. Lett. 24, 1832 (1999).

[7] G. Compagno and F. Persico, Phys. Rev. A 22, 2108 (1980).

[8] E. T. Jaynes and F. W. Cummings, Proc. IEEE 51, 89 (1963); C. Cohen-Tannoudji, Cargese Lect. Phys. 2, 347 (1968).
[9] C. Cohen-Tannoudji, in Frontiers in Laser Spectroscopy, edited by R. Balian et al. (North-Holland, Amsterdam, 1977).

[10] M. Alexanian and S. K. Bose, Phys. Rev. A 52, 2218 (1995); Y. Wu, ibid. 54, 1586 (1996).

[11] D. A. Holm and M. Sargent, III, Opt. Lett. 10, 405 (1985).

[12] D. A. Hutchinson and C. Mavroyannis, Opt. Commun. 38, 314 (1981); C. Mavroyannis, ibid. 26, 453 (1978).

[13] T. H. Cheung, Chin. J. Phys. (Taipei) 21, 18 (1983).

[14] S. K. Basu, T. Pramila, and D. Kanjilal, Opt. Commun. 45, 43 (1983).

[15] Z. Chen and H. Freedhoff, J. Phys. B 24, 1935 (1991).

[16] M. Alexanian, S. K. Bose, and L. Chow, J. Mod. Opt. 45, 2519 (1998); Y. Wu and X. Yang, Phys. Rev. A 56, 2443 (1997).

[17] G. Compagno, R. Passante, and F. Persico, Atom-Field Interactions and Dressed Atoms (Cambridge University Press, 
Cambridge, England, 1995).

[18] C. Cohen-Tannoudji, Atoms in Electromagnetic Fields, 2nd ed. (World Scientific, Singapore, 2004), p. 238.

[19] C. Cohen-Tannoudji and S. Reynaud, J. Phys. B 10, 345 (1977)

[20] C. Leonardi, F. Persico, and G. Vetri, Riv. Nuovo Cimento 9,
1 (1986).

[21] W. Heitler, The Quantum Theory of Radiation, 3rd ed. (Dover Publications, New York, 1984).

[22] B. R. Mollow, Phys. Rev. A 12, 1919 (1975).

[23] Murray Sargent III, D. A. Holm, and M. S. Zubairy, Phys. Rev. A 31, 3112 (1985). 